\title{
El enfoque Ciencia Tecnología Sociedad desde el currículo propio: Género Salud y Sexualidad. Su intencionalidad formativa en la educación superior The Science Technology Society approach from the own curriculum: Gender Health and Sexuality. Her formative intention in higher education
}

\author{
MSc. Liuvys Angarica García ${ }^{1}$ \\ langarica@ucf.edu.cu
}

Recibido: 1/12/2019; Aceptado: 4/3/2020

\begin{abstract}
RESUMEN
El trabajo que se presenta aporta los sucesos más preponderantes en la historia de la sexualidad como fenómeno social y su vinculación con los estudios científicos realizados, respondiendo a las condicionantes en que este se comprende desde la antigüedad hasta los momentos actuales. Por otra parte, se abordan las consecuencias de la sexualidad desde el punto vista educativa a través del tratamiento de un enfoque Ciencia-Tecnología-Sociedad (CTS), desde la asignatura Género Salud y Sexualidad. Para ello, el trabajo se centra además en una metodología con enfoque ceteísta que accede a una mejor implementación de la asignatura, para los estudiantes de la carrera Licenciatura en Educación, Biología, de la facultad de Ciencias Agraria de la UCF Carlos Rafael Rodríguez. La metodología asume los procedimientos para cada una de las fases que llevan a la incorporación en los contenidos y los métodos, los adelantos de la ciencia y la tecnología en la sexualidad que repercuten en la sociedad.
\end{abstract}

Palabras clave: enfoque CTS, salud y sexualidad, currículo

\section{ABSTRACT}

The work that is presented contributes the most preponderant events in the history of sexuality as a social phenomenon and its link with the scientific studies carried

\footnotetext{
${ }^{1}$ Universidad Carlos Rafael Rodríguez, Cienfuegos. Cuba.
} 


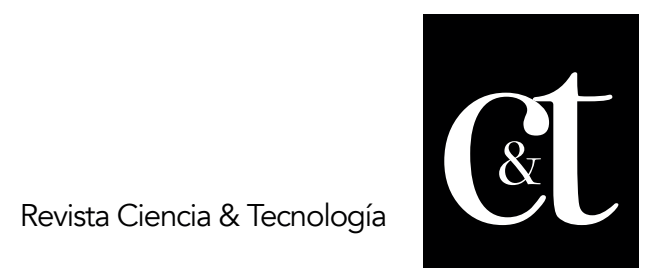

No. 26, 30 de abril de 2020

ISSN impreso: 1390 - 6321

ISSN online: 2661 - 6734

out, responding to the conditions in which it is understood from antiquity to current times. On the other hand, the consequences of sexuality are addressed from the educational point of view through the treatment of a Science-Technology-Society (CTS) approach, from the subject Gender Health and Sexuality. For this, the work is also focused on a methodology with a ceteista approach that accesses a better implementation of the subject, for the students of the Bachelor's Degree in Education, Biology, of the Faculty of Agricultural Sciences of the UCF Carlos Rafael Rodríguez. The methodology assumes the procedures for each of the phases that lead to the incorporation in the contents and methods, the advances of science and technology in sexuality that have an impact on society.

Keywords: CTS approach, health and sexuality, curriculum

\section{Introducción}

Abordar el enfoque Ciencia-Tecnología-Sociedad implica detenerse en las acepciones que conforman el término y entender la tríada dialéctica que sustenta dichos estudios. La ciencia es vista como un fenómeno complejo que se revela en sus múltiples conexiones con la sociedad, es decir, como una forma específica de la actividad social dirigida a la producción, distribución y aplicación de los conocimientos acerca de las leyes de la naturaleza y de la sociedad.

Por su parte la tecnología, según Núñez (1999), es más que un resultado científico, único e inexorable, y debe ser vista como un proceso social, una práctica, que integra factores psicológicos, sociales, económicos, políticos y culturales; siempre influido por valores e intereses.

En sus orígenes, la tecnología se desarrolla a partir de la técnica, hoy es fruto del desarrollo científico; es la ciencia quien proporciona conocimientos fundamentales para múltiples ramas de la tecnología y ella a su vez en su desarrollo genera nuevos conocimientos científicos.

Por su parte, la ciencia de hoy día se orienta cada vez más a fomentar el desarrollo tecnológico y con éste la innovación; su realización y desarrollo no es posible sin la utilización de los modernos recursos creados por la tecnología (computadoras, potentes microscopios y telescopios, satélites, nuevos materiales, entre otras).

La ciencia y la tecnología no se pueden estudiar fuera del contexto social en el que se manifiestan. Entre la ciencia y la tecnología existe un claro estado de simbiosis; en otras palabras, conviven en beneficio mutuo. Aunque el efecto de ambas actuando conjuntamente es infinitamente superior a la suma de los efectos de cada una actuando por separado.

Hoy en día, la tecnología es parte del sistema de vida de todas las sociedades. La ciencia y la tecnología se están sumando a la voluntad social y política de las sociedades de controlar sus propios destinos, sus medios y el poder de hacerlo. Ellas están proporcionando a la sociedad una amplia variedad de opciones en cuanto a lo que podría ser el destino de la humanidad. Y por su parte la sociedad genera nuevos retos a la ciencia y la tecnología.

Y es que, en la medida en que la sociedad asuma una correcta interpretación 


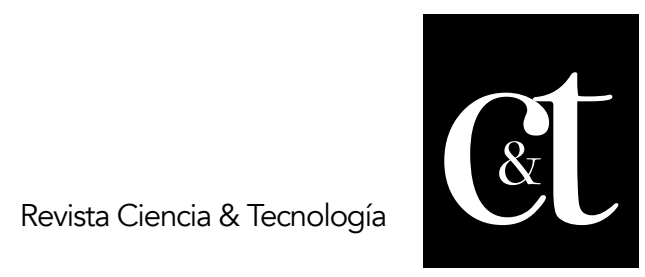

No. 26, 30 de abril de 2020

ISSN impreso: 1390 - 6321

ISSN online: 2661 - 6734

conceptual de la ciencia y la tecnología, a partir de enfatizar en su naturaleza social, se comprenderán mejor los impactos económico, cultural, político y de todo orden que tienen la ciencia y la tecnología a escala global y permitirá una actuación consecuente al respecto.

Lo antes explicado no se puede lograr al margen de la educación como proceso, por lo que es innegable la responsabilidad que tienen los sistemas educacionales, ellos deben adecuar su desarrollo conforme a la dinámica que se establece entre ciencia, tecnología y sociedad e intentar relacionar la ciencia y la tecnología con el medio natural y social, a partir de recuperar sus aspectos sociohistóricos, mostrando una visión más contextualizada de la ciencia y su aspecto motivador.

Todo esto facilitará a los estudiantes valorar el desarrollo científico- tecnológico, su utilidad, comprender cómo ha evolucionado y qué implicaciones y consecuencias sociales, políticas, culturales, ambientales, para la salud y laborales traen a la sociedad.

Entendiendo estos aspectos nos adentramos en la sexualidad visto como un fenómeno social que encuentra desde sus condicionantes sociales la explicación de cómo ha evolucionado históricamente y al analizar las consecuencias que desde el punto de vista político, económico, ético, estético tiene en la actualidad. A partir de una metodología que se presenta como parte de la asignatura Género Salud y Sexualidad.

\section{Desarrollo}

\section{La sexualidad desde la antigüedad hasta nuestros días}

Desde la propia existencia de la humanidad, los instintos sexuales se encuentran entre los más fuertes de nuestros impulsos básicos, además de perpetuar la especie. Lo que significa que esta surge primero como un fenómeno biológico.

En la antigüedad las manifestaciones sexuales se reducían al sexo, el cual era considerado en el matrimonio como una obligación y una alegría que celebra la relación espiritual, además de física entre la mujer y el hombre. En otras civilizaciones existía la práctica del hedonismo, homosexualidad, bisexualidad, el aborto, así como la práctica del ascetismo y las penalidades física, como forma de asegurar la vida después de la muerte.

En la Edad Media el cristianismo cobró fuerzas, por lo que la iglesia asumió la jurisdicción del matrimonio, además de establecer reglas acerca de la conducta sexual. El sexo era considerado un asunto grave y pecaminoso. La actitud religiosa de soltería y ascetismo era la forma de vida que se proclamaba como más deseable, sin embargo, la realidad era otra, hasta para muchos casos de la vida de sacerdotes y monjas que se alejaba de la castidad y el celibato.

En la época del Renacimiento el cuerpo humano, la sexualidad y lo referente a cuestiones sexuales fueron de gran interés para los hombres y mujeres que comienzan a explorar su mundo inmediato y experimentarlo en forma científica en plantas y animales, además de interesarse por el estudio de la humanidad. Las costumbres sexuales siguieron otro proceso. Se establece dentro del Antiguo Testamento de forma estricta al sexo dentro del matrimonio y la celebración del vínculo matrimonial. 


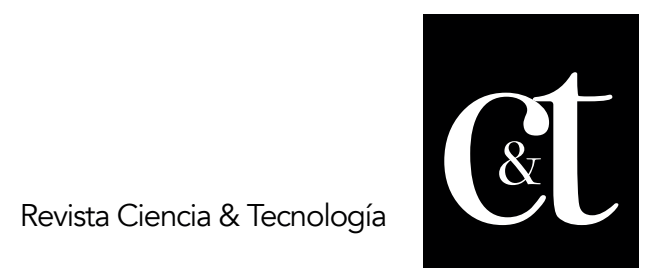

No. 26, 30 de abril de 2020

ISSN impreso: 1390 - 6321

ISSN online: 2661 - 6734

En los primeros años del siglo XVIII la vida era difícil y permitía pocos adornos para los puritanos. Por lo que la ambición e independencia se fomenta tanto para hombres y mujeres. Lo hombres se ocupaban de la libre empresa y la expansión del país. Sus esposas, por querer conquistar las mismas fronteras, se afirmaban y se hacían independientes.

Esta época se denominó Era Victoriana, que en sentido cronológico se considera equivalente al reinado de la Reina Victoria (1834-1901). La religión ocupó un sitio secundario, la ciencia y los conocimientos intelectuales fue el objeto principal de los estudiosos. Las culturas se fusionaron lo que permitió el intercambio de ideas entre los estudiosos. El sexo se consideraba un desperdicio de energía. Si se exaltaba el impulso sexual, era para considerar esta fuerza en el trabajo y los grandes logros.

En el siglo XIX las personas luchaban por suprimir sus inclinaciones naturales, sus sentimientos humanos esenciales, para estar de acuerdo con la época y la religión. Sin embargo, estas costumbres y tendencias de la era victoriana quedaron atrás, en una sociedad que cambiaba constantemente, que se hacía más compleja y urbana.

Al llegar el siglo XX, con la Primera y Segunda Guerra Mundial, ocurrió un cambio impresionante en los papeles sexuales tradicionales, motivado por la escasez de brazos masculinos. Las mujeres se desempeñaron en las más diversas funciones, tuvieron que afrontar la dirección del hogar y la familia. De esta manera sin proponérselo, comienzan a ejercitar los roles hasta entonces destinados al hombre y prohibidos para su género. Al parecer, comienza la plena libertad sexual de nuestro tiempo.

Durante los años sesenta y setenta se produce una transformación de los valores y modelos de comportamiento sexual, especialmente relacionados a la proyección de la mujer, por lo que se le llamó revolución sexual.

La sociedad en esta etapa más tecnificada y desarrollada descubre en el sexo uno de los recursos más efectivos para vender, para hacer más atractiva la oferta e incrementar la demanda. He aquí el motivo por el cual la sociedad comienza a seguir los tabúes, los perjuicios y las trabas sexuales ancestrales, con un bombardeo de estímulos eróticos, que entre otras consecuencias negativas convierte a la mujer de objeto de reproducción, en objeto sexual. Estos años revelan además el sentir patriarcal de esos tiempos.

En los años siguientes comienza a producirse un conjunto de cambios materiales y espirituales transcendentales en la vida del hombre y la mujer, que abre una nueva era para la sexualidad masculina y femenina. Aun cuando en la sociedad se mantiene la fórmula estereotipada del sexo oficial inherente a las sociedades patriarcales. Se abren nuevas maneras de valorar la conducta, el pensamiento y los sentimientos de ambos sexos.

Lo ante expuesto permite percibir como las sociedades norman y reglamentan de 


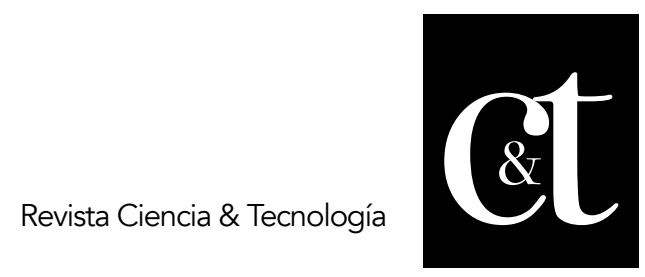

No. 26, 30 de abril de 2020

ISSN impreso: 1390 - 6321

ISSN online: 2661 - 6734

alguna forma las conductas sexuales. El estilo en que lo hace cada grupo social depende de la concepción que se tenga de la sexualidad.

Frente a los impactos de las transformaciones que se despliegan en los escenarios globales, el siglo XXI se redimensiona como nunca, ante el reto de cultivar nuevas formas de vivir, sentir la sexualidad y la pertenencia a un género, en correspondencia con los problemas trascendentales de la sociedad. Se produce una transformación radical de los valores y modelos de comportamientos sexual, especialmente relacionado a la proyección de la mujer.

Surgen nuevas tecnologías en la producción de medicamentos que hacen posible el descenso de la mortalidad infantil, e incremento de una natalidad infantil, fármacos que realza la actividad sexual y los que producen difusión sexual.

Producción de vacunas eficaces en el tratamiento de infecciones de transmisión sexual. La creación tecnológica de anticonceptivos seguros y eficaces que posibilitan la planificación de la reproducción.

Procedimientos quirúrgicos como la histerectomía, mastectomía, vasectomía, y enterostomía que no afecta el funcionamiento sexual humano.

Aparecen tecnologías reproductivas, tales como fertilización in vitro, los bancos de gametos, el implante de embriones que hacen posible la utilización de úteros subrogados, la inseminación, la existencia de técnicas como la terapéutica sexual para el tratamiento de los trastornos sexuales, la clonación y la elección del sexo, estos dos últimos representan nuevos desafíos.

Mucho de los conocimientos científicos de la sexualidad humana de estos tiempos se debe a los estudios realizados en diferentes épocas por investigadores de renombre como el Dr. Masters ginecólogo y obstetra que realizó investigaciones sobre la biología de la sexualidad al igual que Johnson, Havelock, Freud y Kinsey (1894-1956) se destacaron en la investigación sexual en los campos de la sociología y psicología.

La historia de la sexualidad y su vinculación con las ciencias atraviesa un período histórico largo, que pone de manifiesto las inquietudes del hombre por el conocimiento de lo que le rodea, con el que interactúa y del cual depende. Nuestro país no está excepto de lo ante planteado, tal es así, que, como parte indisoluble del proceso de cambios de la conducta sexual de la especie humana, confiere especial atención a la Educación Sexual de las nuevas generaciones.

\section{La Educación Sexual en Cuba: consecuencias educativas}

En tal sentido, desde los primeros años de la revolución se puso en práctica el programa cubano de Educación Sexual. Gestado en sus inicios por la Federación de Mujeres Cubanas, a lo que posterior se sumaron otras instituciones estatales, organismos, organizaciones de masas y sociales del país.

El objetivo fundamental se dirige a la formación integral de niños, niñas, adolescentes y jóvenes, razón por la cual sus contenidos se incluyen en los diversos niveles del currículo escolar y es sometido al perfeccionamiento educacional desarrollado por el Ministerio de Educación en la década del 70 y 80. 


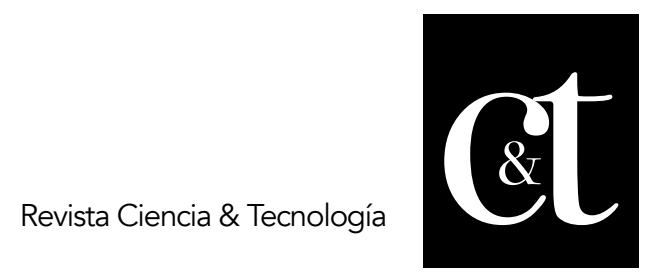

Con la aparición de la epidemia del Sida en la década del 80 se hace necesario fortalecer los procesos de educación para la prevención de la misma, conformándose por el Gobierno el grupo Operativo para el enfrentamiento y lucha contra el Sida (GOPELS). El 4 de enero 1997 en Resolución conjunta con el MINED - MINSAP se oficializó entre otros aspectos la Educación de la Sexualidad, la prevención del virus de Inmunodeficiencia Humana (VIH) y las infecciones de transmisión sexual (ITS) (Castro Alegret, 2007).

En 1999 entra en vigor el Programa Director de Promoción y Educación para la Salud en el Sistema Nacional de Educación. El mismo, establece los ejes temáticos a desarrollar en el currículo escolar, uno de los cuales corresponde a la Educación de la Sexualidad (Gotwald, 2000).

Teniendo en cuenta la relación Ministerial firmada el 1ro de agosto del 2008 por los Ministros de Salud y Educación de América Latina $Y$ el Caribe en conferencia efectuada en México se tomó como acuerdo la implementación de la Educación integral en la sexualidad y la prevención del VIH Sida en el currículo escolar y en la formación y capacitación permanente del personal docente.

El Ministerio de Educación en cumplimiento de estas declaratorias y como parte del proceso de revisión y actualización curricular propone el programa de Educación de la Sexualidad con Enfoque de Género y Derechos Sexuales para su aplicación en el currículo escolar del Ministerio de Educación e Instituciones de formación docentes.

La autora asume el programa para la enseñanza superior propone una metodología con enfoque CTS, que permita la formación integral de los estudiantes, considerados ellos, los futuros educadores de las venideras generaciones en las distintas enseñanzas educacionales.

El sistema educativo cubano por su carácter dialéctico, tiene grandes posibilidades de contribuir a la formación científica, ideológica y humanista de los educandos, partiendo del criterio de que la educación científica no puede limitarse a los conocimientos de las ciencias, sino que estos conocimientos tienen que estar en íntima relación con los problemas sociales y con la búsqueda de soluciones científicas y tecnológicas para enfrentar la dinámica del desarrollo y los cambios que este genera.

Asumiendo lo antes señalado y coincidiendo con Gómez Padilla (2006), es que planteamos la necesidad que tiene el docente de la constante actualización en cuanto al desarrollo de la ciencia y la tecnología, así como los impactos sociales que trae; lo que permitirá nuevas concepciones éticas desde las perspectivas actuales.

En correspondencia con estos planteamientos la asignatura perteneciente al currículo propio Género Salud y Sexualidad incluida en el nuevos plan de estudio (Plan E) para la formación inicial del profesional, de la Carrera Licenciatura en Educación, Biología en el 3er año del curso diurno, integra e interrelaciona aspectos correspondientes a las más diversas esferas de la sexualidad, que resulta imposible comprenderla al margen de las interrelaciones sociales en conjunto con 


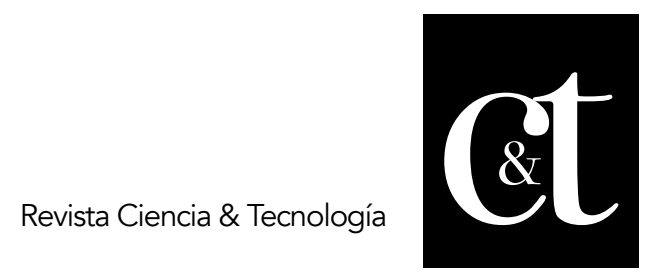

No. 26, 30 de abril de 2020

ISSN impreso: 1390 - 6321

ISSN online: 2661 - 6734

los principios éticos sexuales que la humanidad enfrenta con los descubrimientos científicos en este campo. En efecto permitirá dar cumplimiento a la estrategia curricular de Promoción y Educación para la Salud y los conocimientos bioéticos actuales.

Para lograr la actualización científico - técnica que demanda el proceso de enseñanza- aprendizaje de la asignatura, no es suficiente que aparezca reflejada como objetivo general del programa, no todos los docentes están actualizados en estas temáticas, ni conocen el carácter transformador que las soluciones científico-tecnológicas están potenciando y pueden potenciar para el desarrollo de la sexualidad, formar a estos intelectuales con esta nueva concepción constituye un reto.

\section{Metodología para la asignatura Género, Salud y Sexualidad}

En tal sentido, la incorporación del enfoque CTS desde la asignatura Género Salud y Sexualidad establece una vía para elevar la cultura, e inducir a la actualización del quehacer científico-tecnológico y su aplicación. Es una necesidad en los tiempos en que vivimos; globalizados y complejos, desde el punto de vista, económico, social, político y cultural.

El estudio de la asignatura con enfoque ceteísta tiene gran importancia para el futuro egresado de la carrera de Biología, pues contribuye a su formación integral, los prepara y fortalece para el encuentro libre, pleno y responsable con la sexualidad en correspondencia con sus necesidades y demanda del entorno, los orienta acerca de cómo tomar decisiones personales y trascendentes en problemas de difícil solución, brinda la oportunidad de ampliar su panorama ético y social respecto a la sexualidad. Lo cual repercute en el desarrollo de una sexualidad sana y responsable.

Los docentes que imparte esta asignatura deben comprender que todos y cada uno de los temas propician el enfoque CTS, pues el centro e hilo conductor, lo constituyen la "sexualidad como un hecho universal de vida" (Gonzáles y Castellanos, 2007).

Es por ello que la autora propone una metodología definiéndola en un plano específico como: un conjunto de métodos, procedimientos, técnicas que regulados por determinados requerimientos nos permiten ordenar mejor nuestro pensamiento y nuestro modo de actuación para obtener y descubrir nuevos conocimientos en el estudio de los problemas de la teoría o en la solución de problemas de la práctica (De Armas, 2003).

La metodología para el tratamiento de la asignatura con enfoque CTS consta de tres etapas o fases fundamentales: primera fase: diagnóstico de las consecuencias de la sexualidad, segunda fase: intervención y la tercera fase: proyección, además de un carácter alternativo, participativo y desarrollador.

La primera fase, con el propósito de conocer la realidad del centro y la de los estudiantes, para identificar los problemas existenciales que afectan la vida sexual de los mismos. Así como determinar las necesidades de satisfacción y soluciones a las diversas situaciones. 


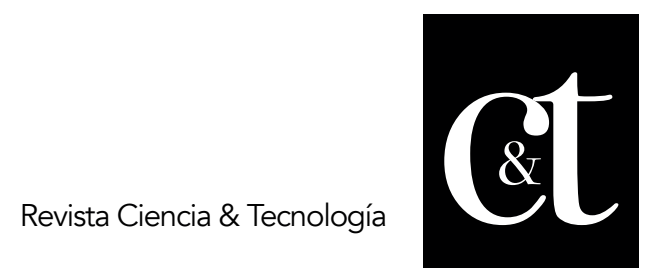

No. 26, 30 de abril de 2020

ISSN impreso: 1390 - 6321

ISSN online: 2661 - 6734

Realizar un estudio del contexto en que se inserta la Universidad, para conocer las costumbres y normas predominantes del entorno, aspectos de la vida cultural comunitaria, y problemas existenciales relacionados con la vida sexual, analizar el los resultados del diagnóstico general integral aplicado a los estudiantes del centro.

En esta fase se debe emplear métodos empíricos como la observación, encuestas, entrevistas, y análisis de documentos que permitan identificar los problemas. Los problemas determinados por la autora existente en la Universidad son: elevado índice de maternidad y paternidad temprana, no se ha logrado en la práctica la equidad de los géneros, existe predominio de la promiscuidad, lo cual no deja en dudas la existencia de infecciones de transmisiones sexuales, las conductas sexuales no siempre son las más adecuadas en nuestros estudiantes, es insuficiente el conocimiento que poseen sobre los temas referente a la sexualidad. Estas situaciones pueden resultar discordantes respecto al estado deseado o modelo educativo trazado por el Gobierno y el Ministerio de Educación y Salud de nuestro país.

Una vez que ha promovido y promueve el ejercicio responsable de la sexualidad, así como las relaciones de equidad entre ambos sexos, legisladas en la constitución.

Para el docente resulta una meta la búsqueda de soluciones los posibles problemas acrecentado en el curso de su labor educativa, encaminada a lograr en los estudiantes la formación general integral con un estilo de vida sano y saludable como se requiere en los momentos actuales a partir del desarrollo científico alcanzado por la humanidad.

Ante los problemas detectados, las necesidades determinadas por la autora para implementar la asignatura con enfoque CTS con los estudiantes de tercer año fueron relacionados a la integración de los conocimientos, actitudes, y valores para enfrentar los desafíos existenciales en cuanto la vida sexual, de pareja, familiar y reproductiva, que enriquezca la calidad de las misma, en cada situación particular.

Lo que implica la transmisión de saberes, experiencias y vivencias en la esfera de la sexualidad por parte del docente.

Una vez que ha identificado los problemas y necesidades que concierne a la Educación Sexual, se procede a la fase de intervención. Aquí se requiere la intervención transformadora y participativa del docente para contribuir a la formación de su estudiante. En esta fase se asumen los objetivos generales y por temas, los contenidos, los métodos, medios, formas de organización y evaluación concebidas para lograr el tránsito del estado actual al deseado. La cual debe contemplar un nivel aceptable de estructuración e integridad, por cuanto garantiza la acción consciente, la intencionalidad en el avance hacia la construcción de nuevos aprendizajes y formación de valores.

Los objetivos propuestos están en función de desarrollar la Educación Sexual como parte de la formación integral del estudiante, sobre bases científicas que 


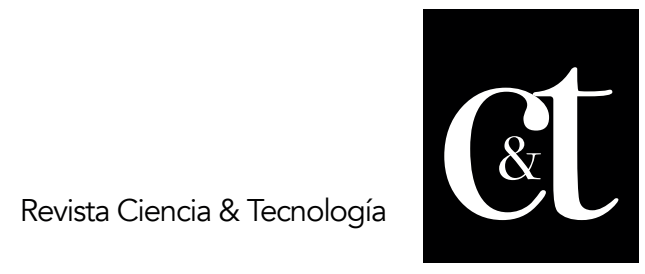

No. 26, 30 de abril de 2020

ISSN impreso: 1390 - 6321

ISSN online: 2661 - 6734

promuevan modos de desarrollo personal y social en diferentes contextos a partir de compararlos desde el punto de vista político, económico, ético, estético, jurídico, y para la salud. En educar sobre los derechos sexuales, como base del desarrollo personal y social, al promover el respeto e igualdad entre personas y la no discriminación, y violencia por razones de género, edad, raza, cultura, identidad u orientación sexual. Así como promover la salud sexual y reproductiva en función de la calidad de vida y prevención de los trastornos de la vida sexual, entre otros.

Los contenidos en función de los objetivos están encaminados al conocimiento de hechos científicos relevantes en las distintas esferas de la sexualidad. Identificarse con su sexo al margen de actitudes y estereotipos sexistas. Aprender a amar y ser amado, a dar y recibir. Comunicarse de modo constructivo con la pareja y demás personas en general. Planear de forma responsable y consciente la familia, la cantidad de hijos y su espaciamiento. Defender y practicar la equidad de género en todas las esferas de la vida. Conocer y defender sus derechos sexuales. Saber sobre la anatomía y fisiología sexual y reproductiva, así cómo alcanzar y preservar la salud sexual y reproductiva (Alvarez, 2004).

Estos conocimientos permiten responder a interrogantes tales como: ¿qué determina el sexo?, ¿cómo se produce el orgasmo?, ¿cuáles son los fármacos que producen difusión sexual y los que realzan la actividad sexual?, ¿cómo se produce la fertilización in vitro?, ¿es ciencia ficción la clonación humana?, ¿cuáles métodos anticonceptivos son más eficaces?, ¿se realizan en Cuba cirugías para transformar el hombre en mujer y viceversa?, ¿quiénes son los transexuales? ¿cuáles son los derechos sexuales y reproductivo?, ¿está legislado el matrimonio entre homosexuales? Es evidente que lo ante expuesto permite adquirir y desarrollar una concepción científica respecto a la sexualidad.

En relación a los métodos que se han de desarrollar en función de los contenidos, estos deben estar basados en situaciones problémica, que propician un aprendizaje desarrollador, tales como: conversación heurística, búsqueda parcial, además del debate, la reflexión. En conjunto se emplean técnicas participativas con el propósito de crear un ambiente cohesionado y cálido, idóneo, para la animación y dinamización del proceso enseñanza-aprendizaje. Entre las cuales pudiéramos citar: Yo soy, el abanico, estoy contento de ser lo que soy, mi compromiso, las que encuentran puntos de contacto con el ciclo de responsabilidades, el método de casos simulados, la discusión plenaria, entre otros, propias del enfoque CTS, Martín, Cerezo, López y Osorio (2009).

Las evaluaciones deberán ser sistemáticas, de forma oral, a partir de seminarios, talleres y una final. Todas centradas en la medición del cumplimiento de los objetivos del programa de asignatura. Luego de la planificación de los contenidos, el docente se encuentra en condiciones de implementar la asignatura con un enfoque CTS además de un carácter alternativo y participativo por todo lo antes expuesto.

Durante el desarrollo de la asignatura debe prevalecer de manera constante la motivación, el interés por saber todo lo concerniente a los temas tratados. El cómo utilizar métodos para implementar sus conocimientos adquiridos en la labor educativa. Confeccionaron medios de enseñanza y técnicas participativas. Hacer 


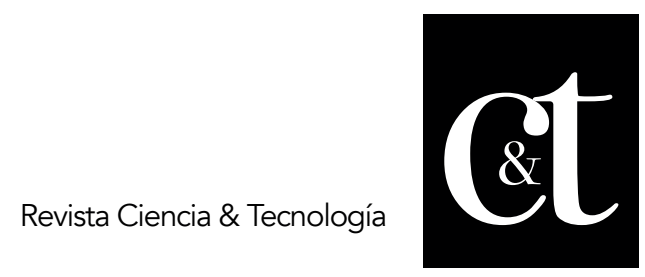

No. 26, 30 de abril de 2020

ISSN impreso: 1390 - 6321

ISSN online: 2661 - 6734

que el proceso de enseñanza aprendizaje sea dinamizado y enriquecedor por cada una de las intervenciones e inquietudes que planteen los estudiantes durante el desarrollo de la asignatura.

El programa debe culminar con una evaluación final donde a los estudiantes le corresponde realizar dramatizaciones y elaborar una clase metodológica desde las diferentes especialidades dándole tratamiento a los temas recibidos por la asignatura que permiten potenciar la Educación Sexual.

Al implementar el programa y con un plazo mayor se consolida la tercera fase de proyección. En esta fase se evalúan los resultados alcanzados por los estudiantes luego de haber recibido la asignatura. Para la misma se tiene en consideración la aplicación de los instrumentos utilizados en la constatación de problemas y necesidades. Los aspectos a considerar son formativos y cognitivos.

Los resultados luego de implementar la asignatura con enfoque CTS deben ser en función de alcanzar una adecuada proyección de sus modos de actuación sobre todo en lo referido a sus manifestaciones sexuales, en ser capaces los estudiantes de darle tratamiento alguno de los temas en las diferentes enseñanzas donde desarrollan la práctica laboral (Giraldo Neira, 1981). Sus manifestaciones y conducta ante la sexualidad se aspiran a que sean adecuadas, reflejo de la educación sexual adquirida durante el proceso (Aller Atucha, 1991). Que las relaciones interpersonales entre los miembros del grupo, sean sin discriminación de sexo, raza, y preferencias sexuales. Sean capases de expresar afectos, sentimientos sin cohibirse, sus expresiones verbales más coherente, claras, lo que demuestra que la comunicación es más asertiva donde prevalezca el respeto y la solidaridad. Alcanzar estos logros hace que la asignatura cumpla sus expectativas.

\section{Conclusiones}

- En el siglo en que vivimos (XXI), somos portadores de toda una historia acumulada de la cual los docentes deben convertirse en gestionadores comprometidos con la ciencia y la tecnología, según la tradición cubana, capaces de promover el análisis y la reflexión, así como la toma de decisiones sobre ética, en los impactos sociales que producen en el ámbito sexual.

- La ciencia y la tecnología son fenómenos sociales que pasan a través de la cultura existente $y$, por tanto, por sus valores, lo que conlleva a una transformación social.

- En particular el problema de las interrelaciones entre ciencia, tecnología, innovación y desarrollo social, con múltiples consecuencias en los campos de la educación y la política científico-tecnológica, merece colocarse en el centro de la atención de los docentes para insertarlo en los programas actuales.

- La implementación de la asignatura en cuestión con tratamiento CTS permite despertar los intereses y motivaciones, lo que contribuye a la Educación Sexual con Enfoque de Género, y Derechos Sexuales en los 78 


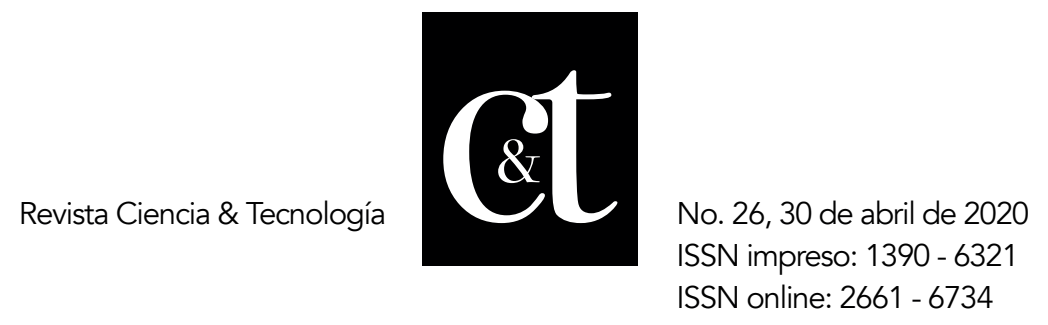

estudiantes de tercer año de la Carrera Licenciatura en Educación, Biología de la UCF.

\section{Referencias bibliográficas}

Aller Atucha, L. (1991). Pedagogía de la sexualidad humana: una aproximación ideológica y metodológica. Editorial Galerma. Buenos Aires.

Alvarez Freire, E. (2004). Fundamentos de anatomía y fisiología humana para maestro. Mariza Rosales Fajardo, Raisa Bernal Cereza. La Habana: Ed. Pueblo y Educación.

Castro Alegret, P. (2007) Educación en la prevención del VIH/SIDA en el Sistema Nacional Escolar. / Maria Antonia Torres Cuesto, Ana Berta López Gómez. La Habana: MINED.

Giraldo Neira, O. (1981). Explorando las sexualidades humanas. México: Editorial Fridas.

González A. y Castellanos B. (2007). Sexualidad, género y familia: una mirada panorámica de su desarrollo en vínculo con el contexto económico y sociocultural. Revista Avanzada Científica Vol. 10 No. 1.

Gotwald, W. (2000). Sexualidad: La experiencia humana. México: Ed. Porrúas.

Katchadourian Herant, A. (1983). La sexualidad humana: un estudio comparativo de su evolución. México: Editorial Fondo de Cultura Económica.

Martín, M., Tedesco, J., López, J., Acevedo, J., Osorio, C. (2009). Educación, Ciencia, Tecnología y Sociedad. Documento de trabajo No 3. OEI. www.oei/cacu.Madrid. España.

Núñez, J. (2013). Material impreso. La Habana, Cuba: OEI, weboei@oei.es.

Núñez, J. (1999). De la Ciencia a la Tecnociencia: pongamos los conceptos en orden. La Habana: Editora Ciencias Sociales.

Núñez, J. (1999). La ciencia y la tecnología como procesos sociales: lo que la educación científica no debería olvidar. La Habana: Editorial Félix Varela. 